\title{
Fraturas do anel pélvico: Estudo epidemiológico
}

\author{
Pelvic ring fractures: Epidemiological study
}

\author{
Alceu Gomes Chueire ${ }^{1}$, Guaracy Carvalho Filho ${ }^{2}$, Antonio Fernando dos Santos ${ }^{3}$, Karen P. Pockel ${ }^{4}$
}

\section{RESUMO}

De fevereiro de 2000 a setembro de 2001, 84 pacientes apresentando fratura do anel pélvico foram avaliados, segundo determinado protocolo aplicado.

Houve predomínio do sexo masculino (67\%), a maioria dos pacientes eram brancos (86\%) e a idade média foi de 37 anos. Quanto ao tipo de acidente, os mais freqüentes foram os relacionados ao trânsito (58\%) - carro, moto e atropelamento - pacientes vítimas de traumas de alta energia.

As fraturas foram classificadas de acordo com Tile ${ }^{(13)}$ em estáveis, em 55\% (fraturas do tipo A); rotacionalmente instáveis, em 30\% (fraturas do tipo B) e rotacional e verticalmente instáveis, em 15\% (fraturas do tipo C). A fratura mais freqüentemente encontrada foi a dos ramos isquiopúbicos.

O tratamento cirúrgico foi realizado em $29 \%$ dos pacientes. Houve necessidade de transfusão sangüínea em $20 \%$ dos pacientes, utilizando-se uma média de cinco unidades de sangue total; exceto dois pacientes $(2,4 \%)$ com lesão arterial intra pélvica que necessitaram mais de 10 unidades de sangue nas primeiras 48 horas.

A mortalidade foi de sete por cento tendo relação significativa com traumas extra pélvicos.

Descritores: Fraturas; Anel pélvico; Epidemiologia

\section{INTRODUÇÃO}

Lesões graves do anel pélvico têm ocorrido com maior freqüência devido aos traumas de alta energia tão prevalentes na sociedade moderna. Nas últimas décadas, muitos avanços foram alcançados tanto na avaliação inicial ao politraumatizado quanto ao tratamento ortopédico dado a tais lesões.

Atualmente, consegue-se uma redução rápida e eficaz das fraturas instáveis da pelve com a utilização de fixadores externos que permitem a mobilização precoce e estabilização imediata do paciente. Associado a isto, os Fundamentos Avançados na Conduta do Trauma (ATLS), aplicados nas Unidades de Pronto-Atendimento e a meIhoria nos sistemas de resgate de pacientes politraumatizados, promoveram uma diminuição da morbi-mortalidade das lesões pélvicas.

\section{SUMMARY}

From February 2000 to September 2001, 84 patients with pelvic fractures were assessed, using a determined protocol.

There were more men (67\%) than women. Most of the patients were white (86\%) and the average was 37 years of age. The most frequent accident was reported to have been traffic accidents (58\%) such as motor vehicle, motorcycle and running over - victims of high-energy injuries.

The pelvic ring fractures were classified according to Tile (13) as stable in 55\% (type A injury), as rotationally unstable in 30\% (type B injury) and as unstable in translation in 15\% (type C injury). The most frequent lesion affecting the pelvic girdle was the isquiopubic bones fracture (transpubic instability).

The overall rate of operative stabilization was $29 \%$. An average of five units of total blood transfusion was required in $20 \%$ of the patients; exception to two $(2,4 \%)$ patients with intrapelvic arterial injury that required more than 10 units of blood in the first 48 hours.

The overall mortality rate was $7 \%$, depending significantly on the associated extrapelvic traumas.

Key words: Fracture; Epidemiology; Pelvic ring.

\section{INTRODUCTION}

Severe lesions of pelvic ring have occurred more frequently due to high-energy traumas so common in modern society. In the last decades, there has been a great deal of improvement in initial evaluation of polytraumatic as well as in orthopedic treatment of these lesions.

Presently, it is possible to decrease unstable pe/vic fractures rapidly and effectively with external fixations that allow early mobilization and immediate stabilization of the patient.

Associated with that, the Advanced Trauma Life Support (ATLS), applied in First Aid Units and the improvement in polytraumatic rescues have promoted a decrease in morbi-mortality of pelvic lesions.

The pelvic ring fractures are little frequent but since they are asso-

\section{Trabalho realizado no Hospital de Base de São José do Rio Preto 1 - Doutor em Medicina, Chefe do Departamento de Ortopedia e Traumatologia da Funfarme-Famerp \\ 2 - Professor-Doutor, Chefe da Disciplina de Ortopedia e Traumatologia da Famerp-Funfarme \\ 3 - Professor Auxiliar de Ensino do Departamento de Ortopedia e Traumatologia da Famerp/Funfarme \\ 4 - Ex-Residente do Departamento de Ortopedia e Traumatologia da Funfarme-Famerp}

Endereço para correspondência : R. Francisco Gigliotti, 546 - São João 15091-280 - S. J. Rio Preto - S.P. - Fone : (17) 226-6412

Trabalho recebido em 09/04/03. Aprovado em 10/02/04
Work performed at Hospital de Base de São José do Rio Preto 1 - PhD Professor in Medicine, Head of the Orthopedics and Traumatology Department of Funfarme-Famerp

2. PhD Professor, Head, Orthopedics and Traumatology Discipline, Famerp-Funfarme

3. Assistant Professor of Orthopedics and Traumatology Department of Famerp-Funfarme,

4. Ex-resident of the Orthopedics and Traumatology Department of Famerp-Funfarme

Address: Rua, Francisco Giglitotti, 546 - São João 15091-280 - S. J. Rio

Preto -S.P. - Phone : (17) 226-6412

E-mail: antoniofsantos@unimedriopreto.com.br 
As fraturas do anel pélvico são pouco freqüentes; porém são as únicas fraturas que por estarem relacionadas a grandes sangramentos retroperitoneais, podem levar a óbito logo após o trauma.

Portanto, em traumas de alta energia, uma lesão pélvica deve sempre ser suspeitada em pacientes politraumatizados graves.

O objetivo do presente estudo foi analisar uma amostra de pacientes apresentando fraturas pélvicas sob abordagem epidemiológica em determinada região. Não foram discutidos os resultados a médio e longo prazo.

\section{MATERIAL E MÉTODOS}

Foi realizado um estudo prospectivo num período de 19 meses (fevereiro de 2000 a setembro de 2001) com pacientes atendidos pelo serviço de Traumato-Ortopedia, apresentando fraturas do anel pélvico.

Ao dar entrada no pronto atendimento hospitalar, todos os pacientes foram inicialmente atendidos pelo grupo da Cirurgia do Trauma e conduzidos, ou transferidos ao grupo da Traumato-Ortopedia. Foram avaliados 84 pacientes; destes, 55 (67\%) eram do sexo masculino e 27 (33\%) do sexo feminino (Gráfico 1). Uma média de idade de 37 anos, variando de três a 88 anos; sendo somente três crianças (Gráfico 2). Setenta e um (86\%) pacientes da raça branca e 11 (14\%) não brancos*

Em todos os pacientes foi realizada uma radiografia de bacia panorâmica ântero-posterior; além de incidências em "inlet" e "outlet". Em alguns pacientes após a internação foi realizada uma Tomografia Computadorizada.

Utilizou-se um protocolo contendo data do acidente, cor, idade, sexo, profissão, tipo de acidente (trânsito, queda de altura, esmagamento e outros), tipo de fratura (utilizouse a classificação de Tile Tabela I) ${ }^{(13)}$, lesões associadas, condutas tomadas e datas das mesmas, complicações imediatas e transfusão sangüínea. Durante a internação foram levantadas desde as complicações mais simples até o óbito, além do método de tratamento utilizado ( redução aberta, fechada ou tratamento conservador ).

Os procedimentos para o tratamento foram: 1) tratamento conservador, 2) redução aberta e fixação interna com placa e parafusos e 3) redução fechada e fixação externa, consistindo de três pinos de schanz colocados percutaneamente em cada íleo, um pela espinha ilíaca ântero superior, outro pelo tubérculo ilíaco (angulados em aproximadamente $45^{\circ}$ um do outro) e um terceiro schanz entre os dois.

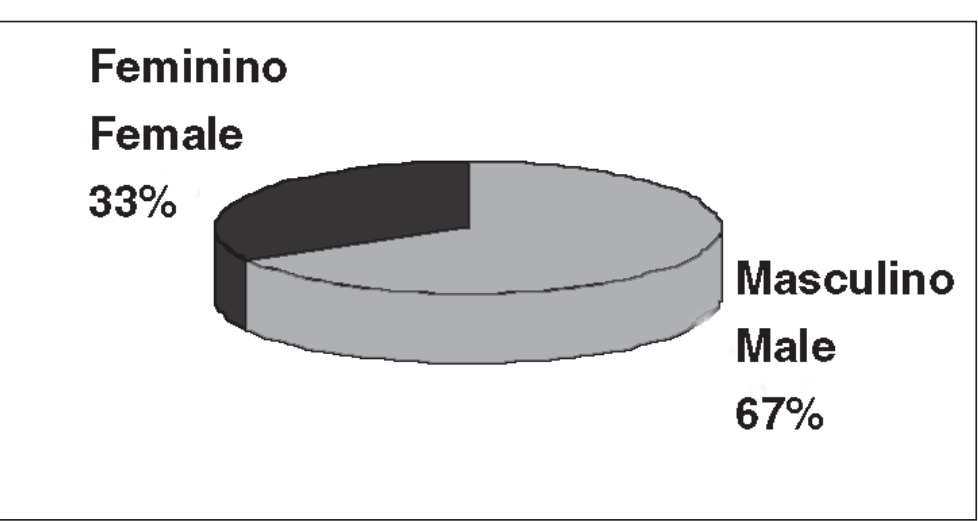

Gráfico 1: Distribuição dos sexos nas fraturas pélvicas.

Graphic 1: Gender distribution among pelvic fractures

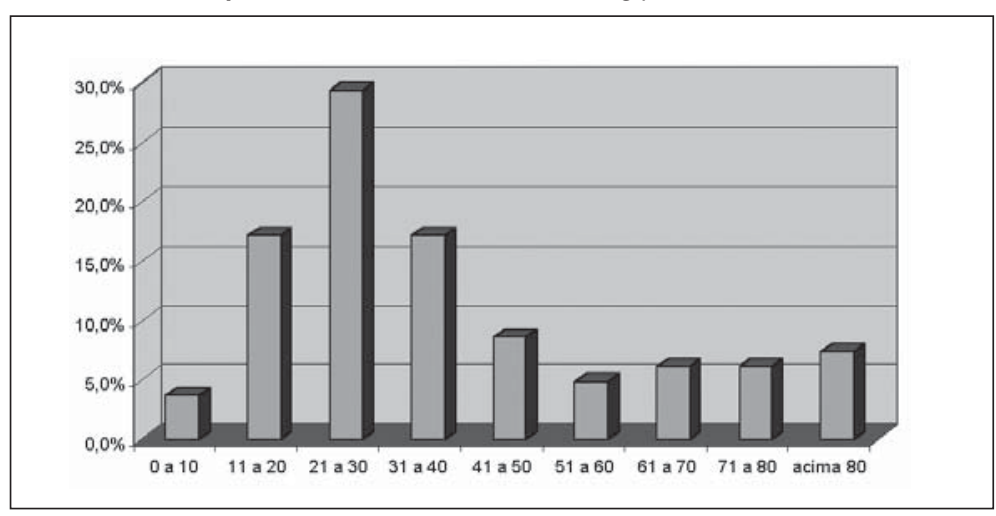

Gráfico 2: Distribuição das idades nas fraturas pélvicas. Graphic 2 - Age distribution in pelvic fractures ciated with large retroperitoneal bleedings they are the only ones which can lead to death soon after the trauma.

Therefore, in high-energy traumas, a pelvic lesion should always be suspected in severe polytraumas.

The aim of this paper was to evaluate a sample of patients with pelvic fractures under the epidemiological approach in a given portion. The medium and long-term results were not discussed.

\section{MATERIALS AND METHODS}

It was performed a prospective study for 19 months (from February 2000 to September 2001) with patients showing pelvic ring fractures in the Traumato-Orthopedics Service.

The Trauma Surgery Group provided the first aids and so the patients were led to the Traumato-Orthopedics Group. 84 patients were evaluated, 55 (67\%) were men and 27 (33\%) were women (Graphic 1).

The average age was 37 years old, ranging from 3 to 88 years old. There were only three children (Graphic 2). 71 (86\%) patients were white and 11 (14\%) were from other races*

Besides the "inlet" and "outlet" occurrences, an anteroposterior panoramic radiograph of the pelvic cavity was performed in all patients. After being admitted to the hospital, some patients underwent a computerized tomograph.

It was used a protocol presenting the date of the accident, color, age, gender, occupation, type of accident (traffic, fall, squash or other), type of fracture (using Tile ${ }^{(13)}$ classification - Table 1) associated lesions, applied procedures and dates, immediate complications and blood transfusion. During hospitalization, all the processes were evaluated from the simplest complications to death, including the methodology of treatment (opening and closed reduction, or conventional treatment)

The procedures for the treatment were: 1) Conventional treatment, 2) opening reduction and internal fixation with plate and screws and 3) closed reduction and external fixation made up of three schanz pins placed percutaneously in each ilium, the first through the anterior superior iliac spine, the second through tuberculum iliaco (angle of $45^{\circ}$ between them) and the third schanz between them.

\section{RESULTS}

In most patients, the pelvic lesion occurred due to traffic accidents (58\%); from these $23 \%$ were car accidents, $19 \%$ were motorcycle accidents and $16 \%$ were running over patients. The second largest group included falls (32\%); of these, $14 \%$ were own height falls and $18 \%$ fell down from very high points (most of the patients were construction workers who fell down from scaffolds). The remaining 10\% were crushed (six $16 \%$ were

Fonte/Source: Funfarme-Famerp 


\begin{tabular}{|cl|}
\hline Tipo / Type A & Fraturas estáveis / Stable Fractures \\
\hline A1 & Fraturas por avulsão - mais comum é na EIAS. / Avulsion Fractures - the most common is the ASIS \\
A2 & Fraturas estáveis da asa do ilíaco ou fraturas pouco deslocadas do anel pélvico. / Wing of ilium stable fractures or less \\
& dislocated pelvic ring fractures. \\
A2.1 & Fraturas isoladas da asa ilíaca. / Isolated wing of ilium fractures. \\
A2.2 & Fraturas do anel pélvico sem deslocamento ou pouco deslocadas. Non-dis/ocated or less dislocated pelvic ring fractures. \\
A2.3 & Lesões isoladas do anel anterior (de quatro pilares). / Anterior ring (of four pillars) isolated lesions. \\
A3 & Fraturas transversas do coccix e do sacro. / Sacrococcyx. transverse fractures \\
A3.1 & Fraturas do coccix ou deslocamento sacrococcígeo. / Coccyx fractures or Sacrococcygeal dislocation \\
A3.2 & Fratura transversa do sacro não deslocada. / Non-dislocated transverse sacro fracture. \\
A3.3 & Fratura transversa do sacro deslocada. / Dislocated transverse sacro fracture. \\
\hline Tipo / Type B & Fraturas parcialmente estáveis./ Partially stable fractures \\
\hline B1 & Lesão em livro aberto (instabilidade rotacional externa). / Open book lesion (external rotatory instability) \\
B2 & Lesão por compressão lateral. / Lateral compression lesion \\
B2.1 & Lesão anterior e posterior ipsilateral. / Anterior and lpsilateral posterior lesion \\
B2.2 & Tipo contralateral (em alça de balde). / Contralateral Type (bucket-handle fracture) \\
B3 & Tipo B bilateral. / Bilateral Type B \\
\hline Tipo / Type C & Fraturas instáveis. / Unstable Fractures \\
\hline C1 & Lesão unilateral. / Unilateral Lesion \\
C2 & Lesão bilateral, sendo um lado B, outro C. / Bilateral Lesion, one side B and other side C. \\
C3 & Lesão bilateral, sendo ambas C. / Bilateral Lesion, both sides C. \\
\hline
\end{tabular}

\section{RESULTADOS}

Na maioria dos pacientes, a lesão pélvica foi devido a acidentes de trânsito (58\%); sendo $23 \%$ acidentes automobilísticos, 19\% motociclísticos e $16 \%$ foram pacientes vítimas de atropelamento em via pública. O segundo maior grupo foi devido a queda (32\%); destes, $14 \%$ foram da própria altura e $18 \%$ caíram de grandes alturas (maioria dos pacientes da construção civil os quais apresentaram queda de andaimes). Os restantes, $10 \%$ sofreram esmagamento $6 \%$ por cento foram esmagados por trator na zona rural), (3\% por cento por caminhão e (1\% por cento soterrado por um muro (Gráfico 3)

Com a análise radiográfica das fraturas a partir da classificação de Tile ( ver Tabela I) foram obtidas: 46 (55\%) fraturas do tipo A, 25 (30\%) do tipo B e 13(15\%) do tipo C.

Das fraturas do tipo $A$, quatro classificaram-se em $A 1$; sendo duas avulsões da espi-

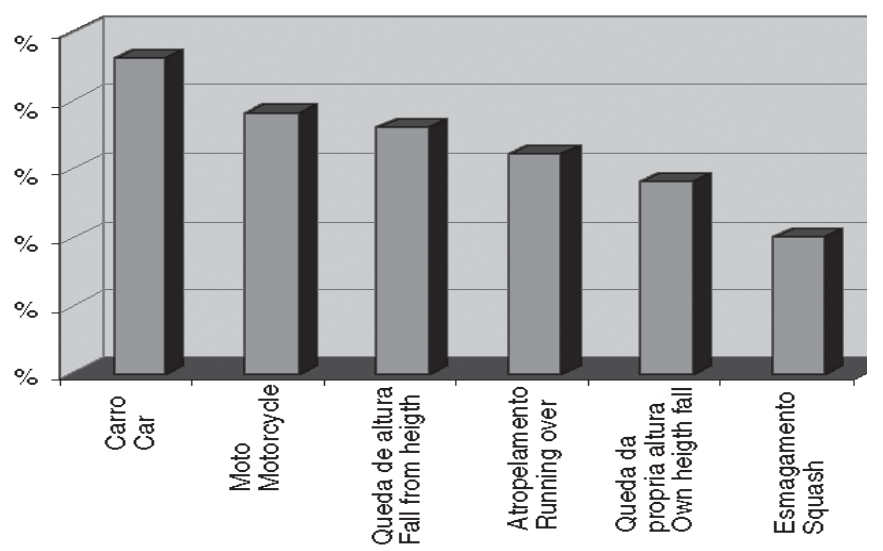

Gráfico 3: Distribuição de freqüência das causas de acidentes. Graphic 3 - Frequency distribution of accident causes.

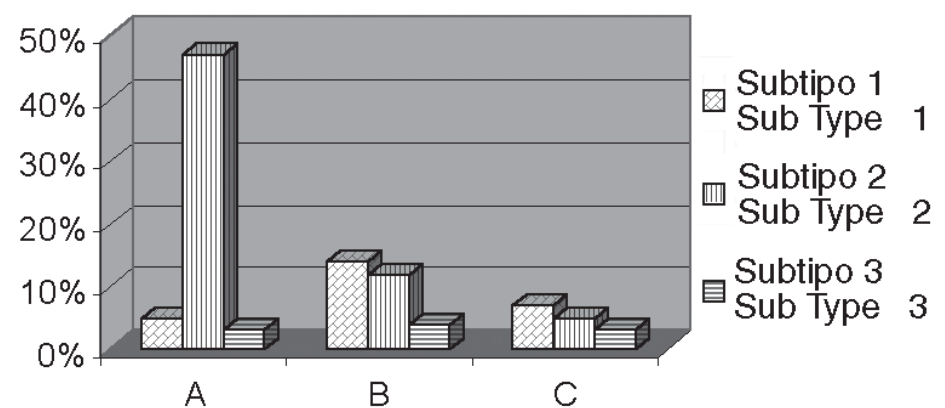

Gráfico 4. Fraturas dos tipos A, B e C classificadas de acordo com Tile. Graphic 4 - Types A, B and C fractures classified according to Tile. crushed by tractors in rural areas) three (3\% were crushed by trucks) and one ( $1 \%$ was buried by a wall) (Graphic 3).

Evaluating the fractures radiographs by using Tile classification (see Table 1) the following figures were obtained: 46 (55\%) type A fractures, 25 (30\%) type $B$ and 13 (15\%) type C.

Among type A fractures, four were classified as A1; two of them were anterior superior iliac spine avulsions, one was of the ramus pubicus in abdominal muscle insertion and the other was an ischial tuberosity. There were ten sub-type A2.1 fractures and only one of them was an open fracture. 28 were sub-types A2.2 and two were sub-type A2.3. In sub-type A 3 fractures, there was one coccygeal fracture and one coccygeal dislocation and the sacral fractures were classified as type $C$.

Of 25 type $B$ fractures, 
nha ilíaca ântero-superior, uma do ramo púbico na inserção do músculo reto abdominal e uma da tuberosidade isquiática. Dez do subtipo A2.1, sendo uma delas a única fratura exposta encontrada. Vinte e oito do subtipo A2.2 e duas do subtipo A2.3. Das fraturas do tipo A3, ocorreu uma fratura e uma luxação coccígea e as fraturas sacrais ocorridas foram incluídas no tipo C.

Das 25 fraturas do tipo B, foram encontradas: 12 do tipo B1, três do tipo B2.1, duas do tipo B2.2 e três do tipo B3. Das fraturas do tipo $C$; seis, quatro e três pacientes apresentaram fraturas dos subtipos C1, C2 e C3, respectivamente (Gráfico 4).

Uma grande quantidade de pacientes (54\%) tiveram outras lesões associadas, sendo que $13(15 \%)$ pacientes necessitaram de laparotomia exploradora. Dois (2\%) destes apresentavam lesões em grandes vasos pélvicos e 11 (13\%), outros traumas abdominais variados. Seis pacientes (7\%) com trauma torácico, 10 (12\%) com traumatismo crânio-encefálico (TCE) e 42 (50\%) apresentavam outras fraturas associadas, sendo 17 (18\%) destas, fraturas de acetábulo.

Dezenove pacientes (23\%) necessitaram de transfusão sangüínea nas primeiras 48 horas. Uma média de cinco unidades de sangue (U) por paciente, variando de duas a dez unidades. Dois pacientes os quais sofreram lesões em grandes vasos (artéria ilíaca), receberam mais de $10 \mathrm{U}$ nas primeiras horas, além de outros derivados de sangue e foram excluídos desta média.

As fraturas estáveis foram tratadas por repouso no leito durante um período variável de três a oito semanas (Figura 1A e B); sendo que apenas uma delas, uma fratura exposta da asa do ilíaco, foi fixada na urgência por redução aberta e fixação interna (RAFI) por fios de Kirschner.

Das 12 fraturas em livro aberto, tipo B1 (Figura 2A e B), em quatro delas foi utilizado fixador externo na admissão e duas foram fixadas por RAFI com placas de auto compressão. Estas apresentavam abertura da sínfise púbica superior ou igual a $2,5 \mathrm{~cm}^{(13)}$.

Das 10 fraturas do subtipo B2, apenas duas foram tratadas cirurgicamente (uma fixada por fixador externo e uma com RAFI). Das três do subtipo B3, duas foram tratadas por fixador externo.

Todas as fraturas do subtipo $\mathrm{C} 1$ foram tratadas cirurgicamente (quatro na admissão por fixador externo e duas durante a internação por RAFI).

As fraturas do subtipo C2 e C3 foram todas fixadas na urgência com fixador externo, por ser uma das montagens mais seguras e rápidas dentre as descritas por outros autores ${ }^{(6)}$ ( Figura $3-A, B, C$ ).

Como complicações imediatas foi encontrado: uma síndrome compartimental em coxa que resultou em amputação, três tromboses venosas profundas (TVP) as quais foram tratadas clinicamente, uma embolia pulmonar e um caso de neuropraxia do nervo ciático tratado conservadoramente.

Ocorreram seis óbitos (7\%); porém todos apresentando uma ou mais lesões associadas de tórax, abdome ou crânio.

A tabela 2 mostra uma avaliação comparativa dos subtipos das fraturas e suas porcentagens relativas quanto ao número de pacientes transfundidos, presença de lesões associadas, tratamento cirúrgico e mortalidade.

\section{DISCUSSÃO}

A maioria das fraturas pélvicas ocorreram em homens, brancos, com idade média de 37 anos e vítimas de acidentes de trânsito.

A raridade das fraturas pélvicas em crianças (três pacientes ou $3,5 \%)$ é explicado pela presença dos componentes cartilaginosos (sínfise pubiana, cartilagem trirradiada e cartilagem das articulações there were: 12 type B1, three type B2.1, two type B2.2 and three type B3. Among type $C$ fractures, there were six sub-type $C 1$, four sub-type C2 and three sub-type C3 (Graphic 4).

A large amount of patients (54\%) had other associated lesions; 13 (15\%) patients underwent exploratory laparotomy.

Two of them (2\%) had large pelvic vessels lesions and 11 (13\%) other varied abdominal traumas. Six patients (7\%) with thoracic trauma, 10 (12\%) with cranioencephalic traumas (CET) and 42 (50\%) presented other associated fractures including 17 (18\%) acetabulum fractures.

19 patients (23\%) needed blood transfusion during the first 48 hours. An average of five units of blood $(U)$ were applied per patient, varying from two to ten units.

Two patients with large vessels lesions (iliac artery), received more than $10 \mathrm{U}$ during the first hours, besides other blood derivatives and they were excluded from this average.

The treatment of stable fractures was the bed rest during a variable time, from three to eight weeks (Figure 1 - $A$ and $B$ ); one wing of ilium open fracture was fixed in emergency by using open reduction and internal fixation (ORIF) with Kirschner wires.

From the 12 type B1 (Figures 2 A and B) open book fractures, four were treated with an external fixation during admission and two were fixed by ORIF with autocompression plates. These ones presented pubic symphisis opening superior or equal to $2,5 \mathrm{~cm}^{(13)}$.

From 10 subtype $B 2$ fractures, only two were surgically treated (one by external fixation and the other by ORIF). From 3 subtype B3 fractures, two were treated by external fixation.

All subtype C1 fractures needed surgery (four in admission by external fixation and two during hospitalization by ORIF).

All subtype C2 and C3 fractures were fixed in emergency by external fixation since it is one of the safest and fastest set among the described by other authors ${ }^{(6)}$. (Figure 3-A, B, C).

One compartmental syndrome of the thigh that resulted in amputation, three deep venous thromboses (DVT that were clinically treated, one pulmonary embolism and one sciatic nerve neuropraxia that received a conservative treatment were the immediate complications.

There were six deaths (7\%) and all of them presented one or more associated lesions of the thorax, abdomen or cranium.

Table 2 shows a comparative evaluation of fracture subtypes and their percentage related to the number of transfused patients, occurrence of associated lesions, surgical treatment and mortality.

\section{DISCUSSION}

Most of the pelvic fractures occurred with men, white, mean age of 37 years old and victims of car accidents.

The rarity of the pelvic fractures in children (three patients or 3,5\%) is explained by the presence of cartilage components (pubic symphisis, triradiated cartilage and articular sacroiliac cartilage) that provide certain flexibility and elasticity ${ }^{(11)}$.

Stable type A fractures prevailed in 46 patients (54\%). Of these, the most frequent ones were isquiopubic bones fractures, presenting a significant importance in elder individuals. The second most frequent type of fracture was the pubic symphisis disjunction, found in 12 patients (14\%). The unstable fractures (type C) were the least frequent, occurring in 13 patients (15\%); however, all of them needed surgical treatment and 12 of these patients (92\%) presented associated lesions.

Other authors ${ }^{(13)}$ found approximated numbers showing that type A fractures were the most frequent ranging from 50 to $60 \%$.

Of all patients, 45 (54\%) presented associated lesions. 11 (13\%) 


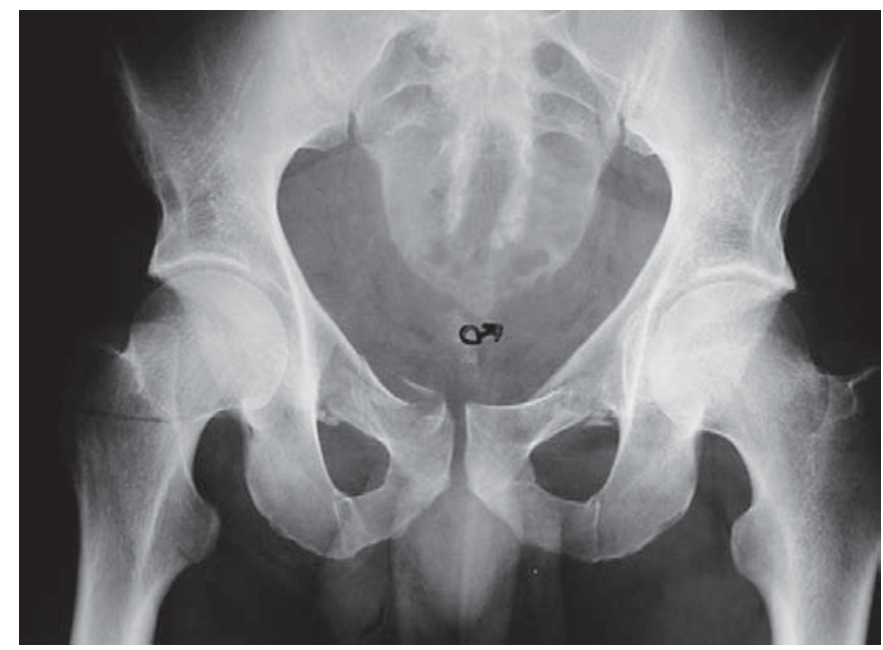

Figura 1A - Paciente masculino, 25 anos de idade, apresentando uma fratura do tipo A2.

Figure 1A - Male patient, 25 years old, presenting type A2 fracture.

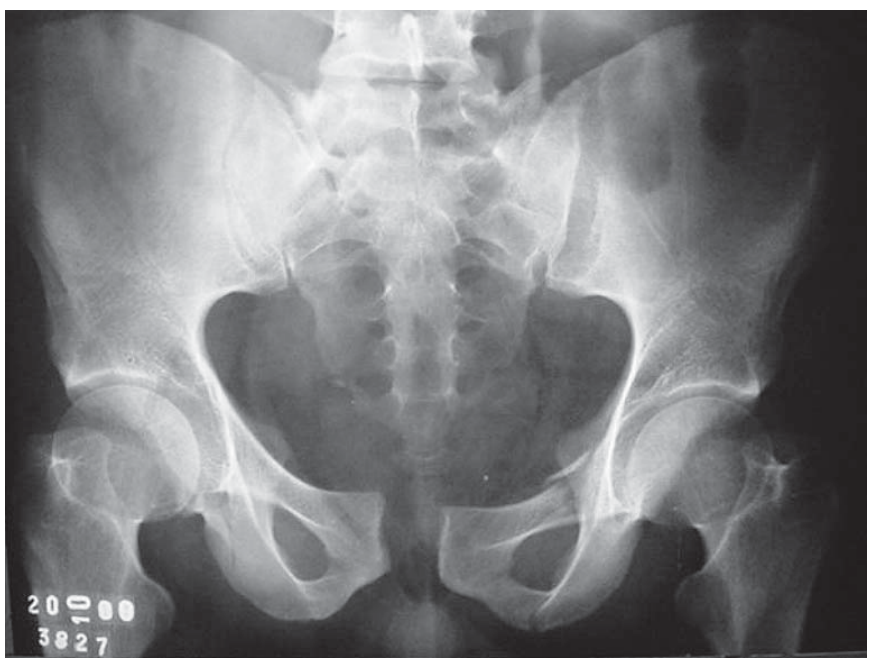

Figura 2A - Paciente masculino, 33 anos de idade, apresentando uma fratura do tipo $B 1$.

Figure $2 A$ - Male patient, 33 years old, presenting type B1 fracture.

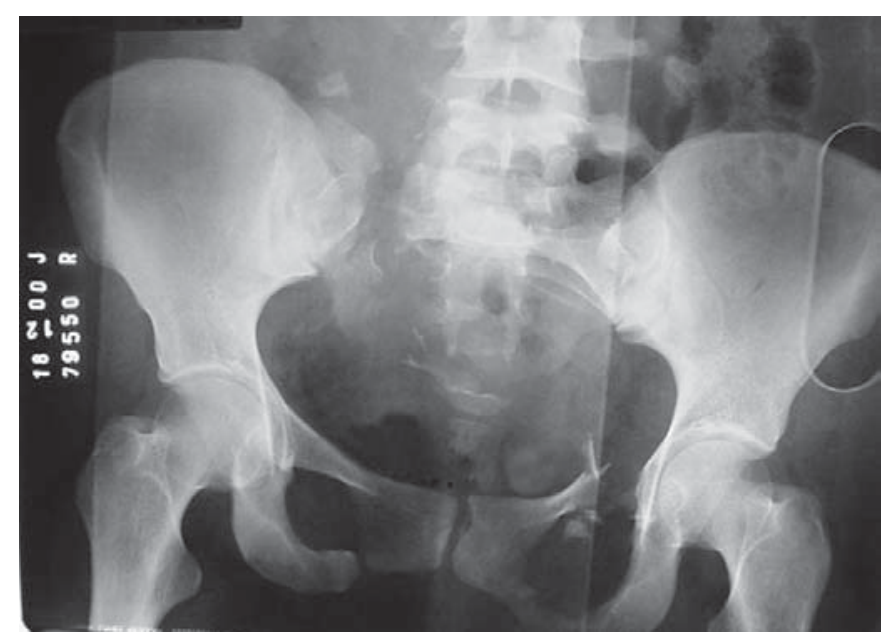

Figura 3A - Paciente feminino, 24 anos, apresentando fratura do tipo C2. Figure 3A - Female patient, 24 years old, presenting type C2 fracture.

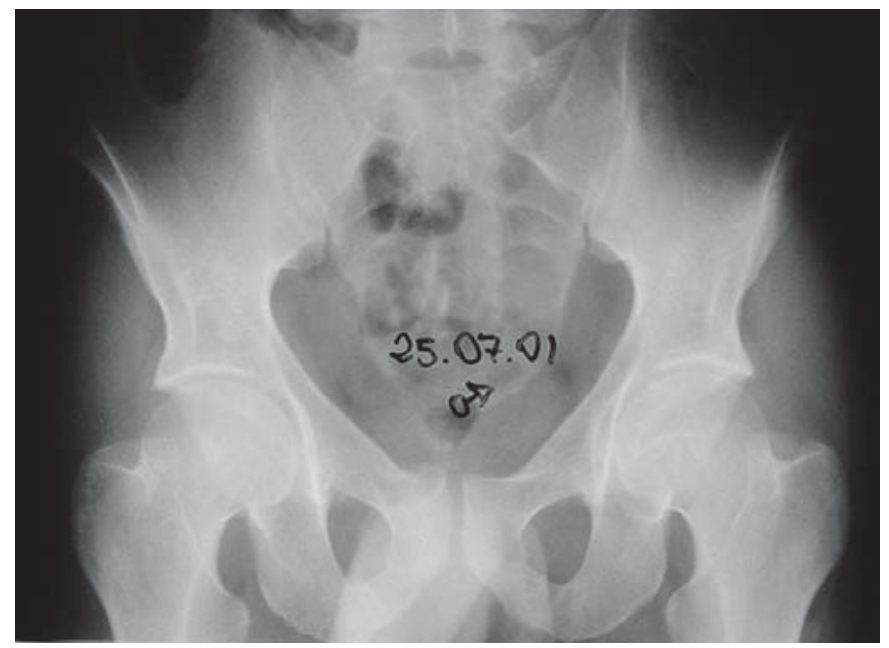

Figura 1B - Cinco meses após tratamento conservador. Figure 1B - Five months after conservative treatment.

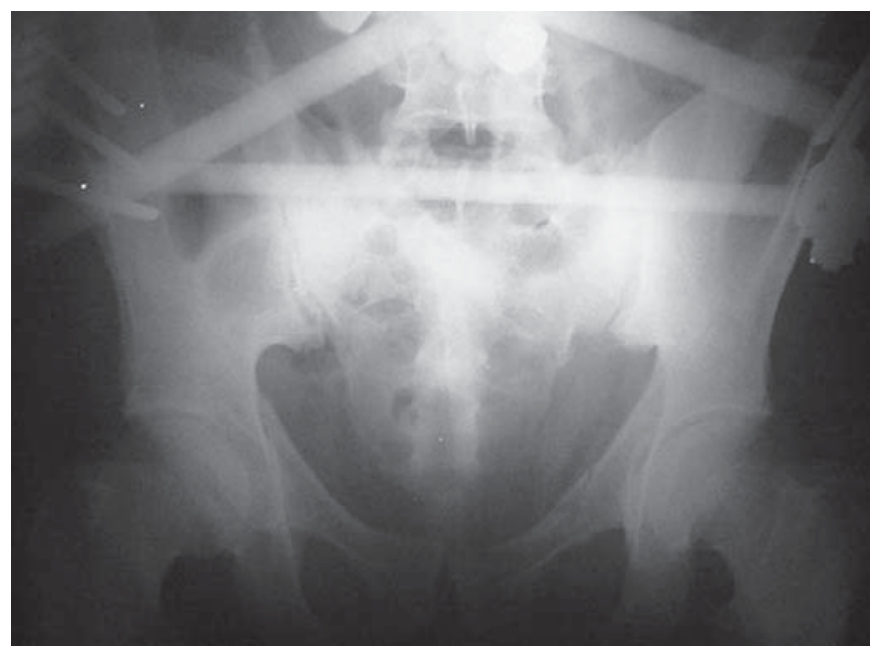

Figura 2B - Pós operatório imediato mostrando redução anatômica com fixador externo.

Figure 2B - Immediate postoperative radiograph showing anatomic reduction using an external fixation.

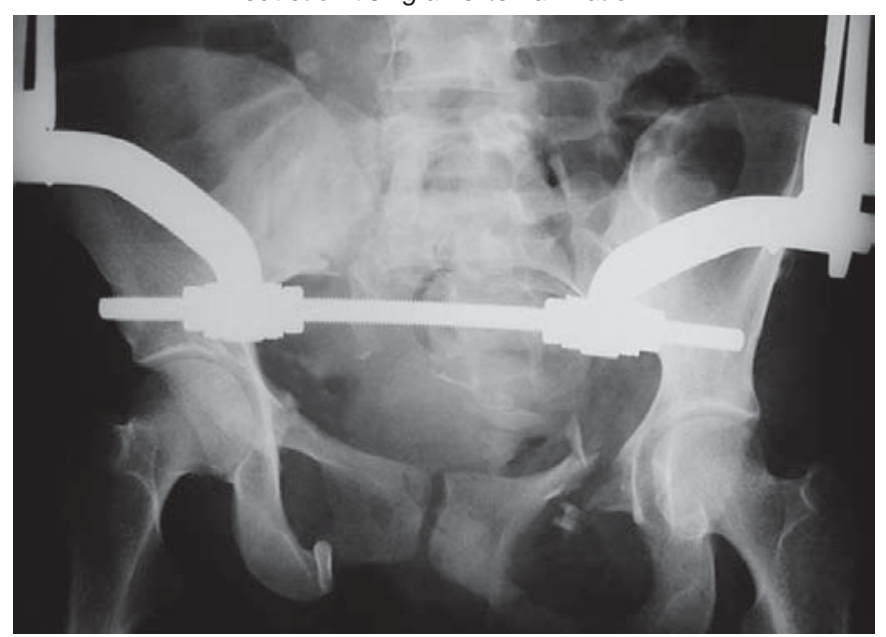

Figura 3B - Pós operatório com fixador externo. Figure $\mathbf{3 B}$ - Reduction using an external fixation. 
sacroilíacas), que dão certa flexibilidade e elasticidade ${ }^{(11)}$.

Fraturas estáveis do tipo A foram as mais prevalentes, 46 pacientes (54\%). Destas, as mais freqüentes foram as fraturas dos ramos isquiopúbicos, apresentando significativa importância em indivíduos mais idosos. O segundo tipo mais encontrado foi a disjunção da sínfise púbica, encontrada em 12 pacientes (14\%). As fraturas instáveis (tipo C) foram as menos freqüentes, ocorreram em 13 pacientes $(15 \%)$; porém sua totalidade necessitou de tratamento cirúrgico e 12 destes pacientes (92\%) apresentaram lesões associadas. Números aproximados foram encontrados por outros autores ${ }^{(13)}$, sendo as fraturas do tipo $A$ as mais freqüentes variando de 50 a $60 \%$.

Da totalidade de pacientes, 45 (54\%) apresentaram lesões associadas. Onze pacientes $(13 \%)$ foram submetidos à laparotomia exploradora, seis $(7 \%)$ apresentaram trauma torácico, dez (12\%) TCE e dezessete pacientes (18\%) apresentaram fraturas de acetábulo associadas. Percentuais aproximados são relatados por outros autores ${ }^{(5)}$.

As taxas de mortalidade encontradas na literatura vão de 4,4 a $30 \%$. Em nosso estudo foi de sete por cento sendo que a complexidade do trauma pélvico teve efeito direto nesta taxa

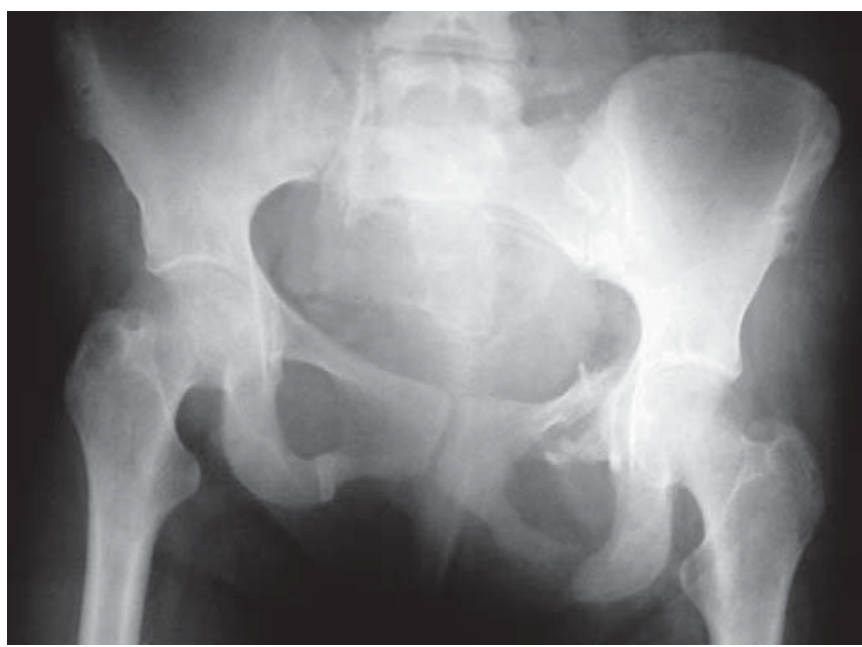

Figura 3C - (C) Evolução após 5 meses mostrando consolidação da fratura.

Figura $3 C$ - (C) Evolution after 5 months showing fracture consolidation underwent exploratory laparotomy, six (7\%) presented thoracic trauma, ten (12\%) CET and 17 patients (18\%) showed associated acetabulum fractures. Other authors ${ }^{(5)}$ report approximated percentages.

The mortality rates found in literature range from 4,4 to $30 \%$. In our study, they reached $7 \%$ and the complexity of the pelvic trauma had a direct influence in this rate. Gänsslen et al. ${ }^{(3)}$ report that the mortality rate was $10,8 \%$ for patients without soft tissues lesions and increased to $31,5 \%$ for complex traumas.

Of the patients who died, three (50\%) presented a CET in some degree. Muir et al. ${ }^{(8)}$ showed in their study a significantly higher mortality rate among those patients who presented pelvic fractures, CET or cervical lesion

Bleedings were con-

\begin{tabular}{|c|c|c|c|c|c|}
\hline Classificação & $\mathbf{N}^{\circ}$ pacientes & $\begin{array}{c}\text { Transfusão } \\
\text { sangüínea (\%) }\end{array}$ & $\begin{array}{c}\text { Lesões } \\
\text { associadas (\%) }\end{array}$ & $\begin{array}{c}\text { Tratamento } \\
\text { cirúrgico (\%) }\end{array}$ & $\begin{array}{c}\text { Mortalidade } \\
\text { (\%) }\end{array}$ \\
\hline Classification & No patients & $\begin{array}{c}\text { Blood } \\
\text { transfusion (\%) }\end{array}$ & $\begin{array}{c}\text { Associated } \\
\text { injuries (\%) }\end{array}$ & $\begin{array}{c}\text { Surgical } \\
\text { treatment (\%) }\end{array}$ & $\begin{array}{c}\text { Mortality } \\
\text { (\%) }\end{array}$ \\
\hline A1 & 4 & & $2(50 \%)$ & & \\
A2 & 40 & $6(15 \%)$ & $18(45 \%)$ & $1(2,5 \%)$ & $1(2,5 \%)$ \\
A3 & 2 & & & & \\
B1 & 12 & $2(16 \%)$ & $4(33 \%)$ & $6(50 \%)$ & \\
B2 & 10 & $2(20 \%)$ & $7(70 \%)$ & $2(20 \%)$ & $3(20 \%)$ \\
B3 & 3 & $2(66 \%)$ & $2(66 \%)$ & $2(66 \%)$ & $1(33 \%)$ \\
C1 & 6 & $3(50 \%)$ & $6(100 \%)$ & $6(100 \%)$ & \\
C2 & 4 & $1(25 \%)$ & $3(75 \%)$ & $4(100 \%)$ & \\
C3 & 3 & $3(100 \%)$ & $3(100 \%)$ & $3(100 \%)$ & $1(33 \%)$ \\
\hline
\end{tabular}

Tabela 2 - Distribuição das fraturas pélvicas em relação à freqüência, transfusão sangüínea, lesões associadas, tratamento cirúrgico e mortalidade.

Table 2 - Pelvic fractures distribution related to frequency, blood transfusion, associated injuries, surgical treatment and mortality. sidered leaders in death cases $^{(7)}$ and the bleeding source was usually regarded to the pelvic fracture (arterial or venous lesions, spongy bone and adjacent tissues lesions) or a combination of intraperitoneal, thoracic or long bones traumas $^{(3)}$. Besides being a direct reason for deaths, the hemodynamic instability is constant in complex pelvic cavity fractures. Of the evaluated patients, 23\% underwent to blood transfusion using an average of 5 units of blood (U)

Cryer et al. (2) report that 36 to $55 \%$ of types $B$

Gänsslen et al. ${ }^{(3)}$ relata que para pacientes sem lesão de tecidos moles, a taxa de mortalidade foi de $10,8 \%$, e em casos de traumas complexos esta taxa subiu para $31,5 \%$.

Dos pacientes que foram a óbito, três (50\%) apresentavam algum grau de TCE. Muir et al..$^{(8)}$ mostra em seu estudo uma mortalidade significativamente maior naqueles pacientes com fratura pélvica e TCE e em pacientes com lesão cervical.

As hemorragias também foram apontadas como líderes nos casos de óbito(7), sendo a fonte da hemorragia relacionada à própria fratura pélvica (lesões arteriais ou venosas, do osso esponjoso e tecidos adjacentes) ou uma combinação de traumas intra peritoneais, torácicos ou de ossos longos ${ }^{(3)}$. Além de ser causa direta de mortes, a instabilidade hemodinâmica é uma constante nas fraturas complexas de bacia. Dos pacientes avaliados, $23 \%$ foram submetidos às transfusões sangüíneas com uma média de cinco unidades de sangue $(U)$ transfundidas.

Cryer et al. ${ }^{(2)}$ relata que 36 a 55\% das fraturas tipo B e C terão uma lesão intra-abdominal e 6 a 18\% terão uma lesão de artérias pélvicas com grandes perdas sangüíneas. Panetta et al..$^{(9)}$ sugerem o and $C$ fractures will present an intra-abdominal lesion and 6 to $18 \%$ will have pelvic arterial lesions with the loss of large amounts of blood.

Panetta et al. (9) suggest the early use of angiography in all patients that need more than four $U$ during the first 24 hours or more than six $U$ in 48 hours.

In our study, two patients (2\%) presented iliac arterial lesions, surgically fixed by a vascular surgeon.

A patient evolved to a compartmental syndrome of the thigh and another to DVT in the lower limb, next to the lesion site. Both underwent amputation of $1 / 3$ proximal of the thigh. One of the patients died.

Among the immediate complications, the DVT deserves note since it occurred in three patients (3,5\%) added to one case of pulmonary embolism (1,2\%). It is known that patients with complex pelvic lesions have the highest risk of developing DVT and that the risk of embolism in these patients may reach $2 \%$ comparing to the risk of $0,2 \%$ of polytraumatic patients ${ }^{(1)}$.

Regarding surgical treatment, it was found in literature that over $60 \%$ of all the pelvic lesions are stable and don't require stabilization, 
uso precoce da angiografia em todos os pacientes que necessitarem mais que quatro $U$ dentro das primeiras 24 horas ou mais que seis $U$ dentro das 48 horas. Em nossa casuística, dois pacientes (2\%) apresentaram lesões em artérias ilíacas, reparadas cirurgicamente pelo cirurgião vascular. Um paciente evoluiu com Síndrome compartimental em coxa, e um com TVP no membro inferior do lado da lesão. Ambos sofreram amputação em 1/3 proximal de coxa e um foi a óbito.

Dentre as complicações imediatas, merece destaque a TVP, sendo que ocorreu TVP em três pacientes $(3,5 \%)$ e uma embolia pulmonar $(1,2 \%)$. Sabe-se que pacientes com lesões pélvicas complexas têm risco, mais alto de TVP e que o índice de embolia nestes pacientes pode alcançar dois por cento comparado ao índice de $0,2 \%$ de pacientes politraumatizados ${ }^{(1)}$.

Com relação ao tratamento cirúrgico, foi encontrado na literatura, que até mesmo em grandes centros de trauma, mais de $60 \%$ de todas as lesões pélvicas são estáveis e não requerem estabilização e, das que precisam ser estabilizadas cerca de $60 \%$ serão submetidas à fixação externa e $40 \%$ à fixação interna ${ }^{(15)}$. Em nossa casuística obtivemos $29 \%$ de tratamento cirúrgico, sendo $75 \%$ de fixação externa e $25 \%$ de fixação interna.

De acordo com Tile(13), quando a bacia é instável verticalmente, a estabilização com fixador externo é imperfeita e é provável a ocorrência de migração da hemipelve. Destaca inclusive que o tratamento de tais lesões não deveria ser fechado ou aberto; contudo se o tratamento fechado falhar, o autor recomenda que se abra; pois a incapacitação crônica grave é devida ao rompimento do complexo sacroilíaco posterior ou do arco de sustentação de peso da bacia, pseudoartroses e consolidações viciosas; levando à dor crônica, geralmente na região lombar inferior ou sacroilíaca ${ }^{(13)}$.

Outros autores ${ }^{(4,14)}$ também concordam que nos últimos anos a fixação interna tem sido a preferida. Em contraste foi obtido um alto percentual de fixação externa (75\%), o que pode ser explicado pelo curto período de avaliação dos pacientes após a internação. Já que a RAFI só é realizada com o paciente quando estabilizado clinicamente.

\section{CONCLUSÃO}

As fraturas da pelve por trauma de alta energia são lesões graves, com mortalidade significativa e alto índice de lesões associadas.

Nos traumas de alta energia, as fraturas pélvicas sempre devem ser suspeitadas e conduzidas juntamente às demais lesões. O atendimento precoce e a utilização de protocolos como o ATLS dão agilidade e orientam o início de um bom prognóstico.

\section{REFERÊNCIAS BIBLIOGRÁFICAS}

1. Buerger PM, Peoples JB, Lemmon GW et al. Risk of pulmonary emboli in patients with pelvic fractures. Am Surg 59:505-508, 1993.

2. Cryer HM, Miller FB, Evers BM et al. Pelvic fracture classification: correlation with hemorrage. J Trauma 28:973-980, 1988

3. Gänsslen A, Pohleman T, Paul C et al. Epidemiology of pelvic ring injuries. Injury 27:13-20, 1996.

4. Garcia JM, Doblare M, Seral F et al. Three-dimensional finite element analysis of several internal and external pelvis fixations. J Biomech Eng 122:516-522, 2000.

5. McMurtry R, Walton D, Dickinson D et al. Pelvic disruption in the polytraumatized patient: a management protocol. Clin Orthop 151:22-30, 1980.

6. Mears DC, Fu FH. Modern concepts of external skeletal fixation of the pelvis. Clin Orthop 151: 65-72, 1980.

7. Moreno C, Moore EE, Rosenberger A et al: Hemorrhage associated with major pelvic fracture: a multispecialty challenge. J Trauma 26:987-994, 1986.

8. Muir L, Boot D, Gorman DF et al. The epidemiology of pelvic fractures in the even if the patients are in large trauma centers. Of the lesions that need stabilization around 60\% will undergo external fixation and $40 \%$ will undergo internal fixation ${ }^{(15)}$. In our study, $29 \%$ of surgical treatment was obtained, divided into $75 \%$ of external fixation and $25 \%$ of internal fixation.

According to Tile ${ }^{(13)}$, when the pelvic cavity is vertically unstable, the stabilization by external fixation is imperfect and may lead to a migration of the hemipelve. It is emphasized that the treatment of these lesions should not be closed or open; however, if the closed treatment fails, the author suggests the opening. The severe chronicle incapacity originates from the posterior sacroiliac complex or from the sustaining arcus of the pelvic cavity weight, pseudoarthrosis and vicious consolidations leading to chronic pain, generally in the inferior lumbar or sacroiliac region ${ }^{(13)}$.

Other authors ${ }^{(4,14)}$ also agree that in the last few years, the external fixation has been the preferable one. On the other hand, it was obtained a high percentage of external fixation (75\%), which can be explained by the short period of evaluation of patients after hospitalization since the ORFI is only performed when the patient is clinically stable

\section{CONCLUSION}

Pelvic fractures by high-energy traumas are severe lesions, with significant mortality rate and a great number of associated lesions.

In high-energy traumas, pelvic fractures should always be suspected and conducted together with other lesions.

The early attendance and the use of protocols such as the ATLS provide agility and guide the beginning of good prognosis.

Arteriograph in hemodynamically unstable patients is essential and when it is performed early can decrease the morbi-mortality rate in patients with arterial lesions.

An external fixation is very useful in emergency to stabilization of initial hemodynamics.
Mersey Region. Injury 27:199-204, 1996.

9. Panetta T, Sclafani SJA, Goldstein AS et al. Percutaneous transcatheter embolization for massive bleeding from pelvic fractures. J Trauma 25:10211029, 1985

10. Parreira JG, Coimbra R, Rasslan $S$ et al. The role of associated injuries on outcome of blunt trauma patients sustaining pelvic fractures. Injury 31 :677682,2000

11. Quinby WC. Fractures of the pelvic and associated injuries in children. J Pediat Surg 1:353-364, 1966.

12. Rittmeister M, Lindsey RW, Kohl HW. Pelvic fracture among polytrauma decedents. Trauma-based mortality with pelvic fracture: a case series of 74 patients. Arch Orthop Trauma Surg 12:43-49, 2001

13. Tile M. Fraturas da pelve e acetábulo. In:Marvin Tile. Fratura da Pélve e Acetábulo $2^{\mathrm{a}}$ ed. Rio de Janeiro, Revinter, 2002. p.66-101.

14. Trafton P. Pelvic ring injuries. Surg Clin North Am 70:655-669, 1990.

15. Wild J, Hanson G, Tullos H. Unstable fractures of the pelvis treated by external fixation. J Bone Joint Surg Am 64:1010-1019, 1982. 